\title{
Autologous and Nonautologous Blood Transfusion in Patients with Ruptured Ectopic Pregnancy and Severe Blood Loss
}

\author{
Jingxian Huang, Dongquan Qin, Chunlin Gu, Yanjuan Huang, He Ma, Huageng Huang, \\ Fanke Huang, Jiaxin Ruan, and Mei Ling \\ Department of Anesthesiology, The Third Affiliated Hospital of Guangxi Medical University, Nanning, Guangxi 530031, China \\ Correspondence should be addressed to Yanjuan Huang; huangyanjuan66@163.com
}

Received 8 December 2016; Accepted 22 May 2017; Published 14 June 2017

Academic Editor: Jonathan Muraskas

Copyright (c) 2017 Jingxian Huang et al. This is an open access article distributed under the Creative Commons Attribution License, which permits unrestricted use, distribution, and reproduction in any medium, provided the original work is properly cited.

\begin{abstract}
Background. There are some theoretical concerns for the use of intraoperative cell salvage (ICS) in patients with ectopic pregnancy. This study aimed to observe the impact of ICS on the coagulation function and clinical outcomes of patients with ruptured ectopic pregnancy and severe blood loss. Methods. This was a retrospective study of 225 patients with ruptured ectopic pregnancy and severe blood loss treated at the Third Affiliated Hospital of Guangxi Medical University between January 2012 and May 2016. Patients were grouped according to ICS $(n=116)$ and controls $(n=109$, allogenic transfusion and no transfusion). Results. Compared with controls, patients with ICS had shorter hospitalization $(P=0.007)$, lower requirement for allogenic blood products $(P<0.001)$, and higher hemoglobin levels at discharge $(P<0.001)$. There were no complications/ adverse reactions. In the ICS group, hemoglobin at discharge $(-6.5 \%, P=0.002)$ and thrombin time $(-3.7 \%, P=0.002)$ were decreased $24 \mathrm{~h}$ after surgery, while $24 \mathrm{~h}$ APTT was increased $(+4.6 \%, P<0.001)$. In the control group, hemoglobin at discharge $(-16.8 \%, P<0.001)$ was decreased after surgery and $24 \mathrm{~h}$ APTT was increased $(+2.4 \%, P=0.045)$. At discharge, hemoglobin levels were higher in the ICS group $(P<0.001)$. Conclusion. ICS was associated with good clinical outcomes in patients with ruptured ectopic pregnancy and severe blood loss.
\end{abstract}

\section{Introduction}

Intraoperative autologous transfusion is widely used to retransfuse back the patient's own blood [1]. Three techniques are used: (1) intraoperative cell salvage (ICS) (blood is collected during surgery, filtered, washed, and transfused back); (2) preoperative autologous donation (blood is collected and stored before surgery); and (3) acute normovolemic hemodilution (ANH) (blood is collected immediately before surgery, blood volume is restored using fluids, and the blood is reinfused during surgery after major blood loss has ceased, or sooner if indicated). Autologous transfusion is in contrast with allogenic blood transfusion, for which the blood comes from an unrelated or anonymous donor [2]. The main factors in favor of autologous transfusion are the reduction of the risk of blood-borne infections (HIV, hepatitis, prions, etc.) and the protection of a scarce resource $[2,3]$.

Currently, the most commonly used method is cell salvage [2]. After washing, the autologous blood mainly includes packed red blood cells and coagulation components such as plasma, platelets, and coagulation factors have been mostly removed, thus having an adverse effect on the coagulation function of the patients $[4,5]$. Therefore, it is suggested to add coagulation factors to the transfused blood, but doing so raises the costs of the procedure and increases the risk of diseases, especially if blood products are used [2]. Nevertheless, some North American and European studies have demonstrated that normal blood coagulation can be achieved if the concentration of coagulation factors in the transfused blood can be maintained at $20-30 \%$ of the normal levels, without the use of coagulation factors [6]. Coagulopathy may be considered when the volume of transfused blood is $>2 \mathrm{~L}$ and coagulation function tests should then be performed, but there is no recommendation for the use of coagulation factors in all cases [7].

In addition, there are theoretical concerns when autologous transfusion is used in patients with ectopic pregnancy and severe blood loss, because the recovered blood is mixed 
with amniotic fluid and fetal blood, which may cause iatrogenic amniotic fluid embolism and alloimmune hemolysis $[8,9]$.

Therefore, the present study aimed to observe the coagulation function and clinical outcomes of 116 patients with ectopic pregnancy and severe blood loss treated with autologous transfusion. These patients were compared with patients who received allogenic transfusion. The results could provide evidence for the management of blood transfusion in patients with ectopic pregnancy and severe blood loss.

\section{Methods}

2.1. Study Design and Patients. This was a retrospective study of prospectively collected data of consecutively enrolled patients with ruptured ectopic pregnancy and severe blood loss treated at the Third Affiliated Hospital of Guangxi Medical University between January 2012 and May 2016. Starting in July 2013, cell salvage transfusion was used in patients with ectopic pregnancy and severe blood loss, defining a blood loss of $>30 \%$ of total blood volume [10].

Ectopic pregnancy was diagnosed based on medical history and imaging. Using the database, the inclusion criteria were (1) ectopic pregnancy and (2) acute blood loss accounting for $>30 \%$ of total blood volume, which is a nonmandatory indication for transfusion therapy [10]. The exclusion criteria were (1) use of any drugs affecting the coagulation function; (2) primary blood diseases; (3) ischemic heart disease; (4) requirement for cardiopulmonary resuscitation due to severe hemorrhagic shock; or (5) patients with concurrent intrauterine pregnancy and ectopic pregnancy.

Written informed consent was obtained from all patients included in the study and the ethics committee of the Third Affiliated Hospital of Guangxi Medical University approved the study.

2.2. Grouping. During the study period, there were 225 patients with ruptured ectopic pregnancy and acute blood loss. Patients were grouped according to autologous blood transfusion $(n=116)$ and controls $(n=109$, including patients with allogenic transfusion and patients without transfusion).

Each group was divided into subgroups based on the rule that $10 \%$ increase $(400 \mathrm{~mL})$ of blood loss was considered to be a group: blood loss in the N1, N2, N3, N4, N5, and N6 subgroups was $1200-1599 \mathrm{~mL}, 1600-1999 \mathrm{~mL}, 2000-2399 \mathrm{~mL}$, 2400-2799 mL, 2800-3199 mL, and $\geq 3200 \mathrm{~mL}$, respectively. The basis for selecting these subgroups was that, in the present study, the mean weight of the patients was $50 \mathrm{~kg}$ and their mean blood volume was $4000 \mathrm{~mL}(50 \mathrm{~kg} \times 8 \%$ [10]). An acute blood loss of $20-30 \%$ of the blood volume will result in a shock state and blood transfusion therapy can be considered if there is an acute blood loss of $>30 \%$ (i.e., $1200 \mathrm{~mL}$ ) [10]. Therefore, we used a blood loss of $10 \%$ of the blood volume (i.e., $400 \mathrm{~mL}$ ) to divide the subgroups as above.

2.3. Surgical Approach. The surgical approach was recommended by the attending physician according to the patients' specific conditions and the final decisions were made by the patients. Most surgeries (94.2\%) were laparoscopic surgeries; laparotomies were performed because of patients' will or critical condition that immediate surgery was needed. Physicians were more conservative during the January 2012 to January 2013 period, and there were slightly more laparotomies during that period.

Debridement of ectopic pregnancy was performed in all patients. If it was found during the operation that the patients had other diseases that required surgery, the appropriate procedures were undertaken at the same time. Therefore, some patients also received myomectomy/ovarian cystectomy/tubal repair and orthopedic/bilateral tubal ligation. The surgeries were not performed by the same surgeon, but all procedures were performed by operators with surgical qualification.

Intravenous general anesthesia was used, and the narcotic drugs included midazolam, fentanyl, remifentanil, propofol, and cisatracurium besylate. In the perioperative period, limited fluid resuscitation was used, and the major crystalloid solution was sodium lactated Ringer's solution (Sichuan Kelun Pharmaceutical Co., Ltd., China), while hydroxyethyl starch 200/0.5 and physiological saline (Hangzhou Minsheng Pharmaceutical Co., Ltd., China) were used for fluid expansion. If blood pressure was $<90 / 60 \mathrm{mmHg}$, vasopressors such as ephedrine, dopamine, and norepinephrine were used to maintain blood pressure at a normal level.

2.4. Cell Salvage Transfusion. The cell salvage device was an autologous-P3000 blood recovery machine (Beijing Jingjing, Beijing, China). Matching double-lumen suction lines were used to retrieve the blood in the peritoneum. The recovered blood was mixed with heparin $(12,500 \mathrm{U}$ mixed with $500 \mathrm{~mL}$ of $0.9 \%$ sodium chloride) at $200 \mathrm{U}$ of heparin for $100 \mathrm{~mL}$ of blood. Blood was placed into the centrifugal tank for centrifugation and washing. It was pumped into blood recovery bags before being transfused to the patients. The washing solution was $0.9 \%$ sodium chloride and $1000 \mathrm{~mL}$ was usually needed to wash $300 \mathrm{~mL}$ of red blood cells.

A restrictive transfusion strategy was used in all patients. Based on guidelines for perioperative transfusion and adjuvant therapy published by the American Society of Anesthesiologists in 2006 and the guidelines for AABB transfusion in the United States in 2012, red blood cells were infused if hemoglobin levels were $<60-70 \mathrm{~g} / \mathrm{L}$, and transfusion was not needed if hemoglobin levels were $>100 \mathrm{~g} / \mathrm{L}[11,12]$. For patients with hemoglobin levels $60-100 \mathrm{~g} / \mathrm{L}$, whether transfusion was conducted or not was determined by the physicians according to comprehensive factors of the degree of anemia, cardiopulmonary decompensation function, metabolic rate, and age [11, 12]. Starting on July 2013, ICS was used for patients with ectopic pregnancy, and its use was based on the recommendation of the physician and the willingness of the patients.

Coagulation function was monitored according to guidelines [11], using standard laboratory diagnostic tests such as prothrombin time (PT), activated partial thromboplastin time (APTT), thrombin time (TT), and fibrinogen levels. Blood cells were analyzed using a Sysmex ${ }^{\circledR}$ XN-9000 
Automated Hematology Systems (Sysmex, Kobe, Japan). A STA-R evolution automated coagulation device (Stago, Paris, France) was used to measure the coagulation indexes.

Before any surgery with the possibility of a transfusion, the patients or their legal representative was fully informed about the possibility of a transfusion, benefits, risks, and alternative options. The patients or their legal representative signed an informed consent.

In addition to transfusion therapy, patients with postoperative hemoglobin levels of $60-80 \mathrm{~g} / \mathrm{L}$ received auxiliary method of intravenous iron supplement for about 4 days after the operation.

2.5. Data Collection. PT, APTT, international normalized ratio (INR), thrombin time (TT), and fibrinogen levels were collected. Clinical indicators included cure rate, ICU occupancy rate, classification of wound healing, hospitalization duration, operation time, surgery-related complications (postoperative infection and bleeding), amount of blood loss, amount of homologous transfusion/autologous transfusion during hospitalization, transfusion rate, adverse transfusion reactions (allergy, purpura, nonhemolytic febrile reaction, bleeding, and hemolysis), and anemia-related complications (dizziness, palpitation, chest distress, and chest pain) were also collected.

Calculation of the amount of blood loss included the amount of autologous retrieved blood, suction bottles, the sum of intraoperative blood clots, and pads. The calculation of blood clots and pads was based on empirical estimation. The operation time was from the time of surgical incision to the time of surgical suture. The patients were considered cured when their general state was good, vital signs were normal, and the concentration of serum human chorionic gonadotropin was decreased to normal.

2.6. Follow-Up after Discharge. The patients were considered as cured when their general condition was good, with normal vital signs, primary healing of the surgical incisions, and a decline to normal serum levels of HCG. All patients were required to have routine telephone follow-up within 2 weeks after discharge. Patients were required to pay a regular outpatient visit to check the HCG levels. Normally, the HCG levels should show a declining trend until normal. If the HCG levels keep increasing instead of declining and an abnormal HCG result is detected within 2 months postoperatively, rehospitalization for further examination and treatment is required. In the present study, no patients need to return to the hospital because of abnormal HCG results after discharge and there was no patient lost to follow-up.

2.7. Statistical Analysis. Normally distributed continuous data were presented as mean \pm standard deviation and analyzed using Student's $t$-test. Nonnormally distributed continuous data were presented as median (range) and analyzed using the Mann-Whitney test (unpaired data) or Wilcoxon's paired signed rank test (paired data). Categorical data were presented as proportions and analyzed using the Chi-square test. SPSS20.1 (IBM, Armonk, NY, USA) was used for data processing and analysis. Two-sided $P$ values $<0.05$ were considered statistically significant.

\section{Results}

3.1. Characteristics of the Patients. Table 1 presents the characteristics of the patients. All characteristics were similar between the two groups, except the surgical approach $(P=$ $0.007)$, which was because the laparotomy approach and nonautologous transfusion were preferred prior to 2013.

3.2. Operative Characteristics. Table 2 presents the operative characteristics of the patients. Compared with the control group, patients in the ICS group had a slightly shorter hospitalization $(P=0.007)$, lower requirement for allogenic blood products $(P<0.001)$, and higher hemoglobin levels at discharge $(P<0.001)$. There were no complications or adverse reactions in the two groups.

3.3. Coagulation Function. Table 3 presents the coagulation function of the patients. Before surgery, thrombin time was higher in the ICS group $(P<0.001)$. In the ICS group, hemoglobin $(-6.5 \%, P=0.002)$ and thrombin time $(-3.7 \%$, $P=0.002)$ were decreased after surgery, while APTT was increased $(+4.6 \%, P<0.001)$. In the control group, hemoglobin $(-16.8 \%, P<0.001)$ was decreased after surgery and APTT was increased $(+2.4 \%, P=0.045)$. After surgery, hemoglobin levels were higher in the ICS group $(P<0.001)$.

3.4. Subgroup Analysis. Table 4 presents the characteristics of the patients according to the amount of blood loss. Figure 1 shows that the rate of infusion of coagulation components was constantly higher in the control group than in the ICS group across all blood loss subgroups. These data could explain the differences in the coagulation functions according to blood loss, as presented in Table 5. Accordingly, postsurgical PT (median, 14.7 versus $13.9 \mathrm{~s}, P=0.03$ ) and INR (median, 1.14 versus $1.07 \mathrm{~s}, P=0.009$ ) were higher in ICS patients in the 1200-1599-mL blood loss subgroup. Pre- and postsurgical thrombin time were higher in ICS patients in the 2000-2399-mL-blood loss subgroup (before: median, 16.2 versus $14.1 \mathrm{~s}, P<0.001$; after: median, 16.3 versus 15.2, $P=0.001)$. In the ICS group, the 1200-1599-mL blood loss subgroup showed improvements in PT-INR (median, from 1.07 to $1.14, P=0.032$ ) and APTT (median, from 33.7 to $35.8, P=0.017$ ) after transfusion. In the control group, the 1600-1999-mL blood loss subgroup showed improvements in APTT (median, from 33.2 to $36.2, P=0.050$ ) after transfusion.

\section{Discussion}

With the increasing demand for blood transfusion, there are shortages of allogenic blood resources. In addition, there are risks with allogenic transfusion, such as transfusion reactions, infectious diseases, increased mortality, organ dysfunction, and delayed wound healing [2]. There are some theoretical concerns for the use of cell salvage transfusion in patients 
TABLE 1: Baseline characteristics of the patients.

\begin{tabular}{|c|c|c|c|}
\hline & $\operatorname{ICS}(n=116)$ & Controls $(n=109)$ & $P$ \\
\hline Age (years) & $30(17,48)$ & $32(18,45)$ & 0.162 \\
\hline Weight $(\mathrm{kg})$ & $50(40,75)$ & $50(32,80)$ & 0.014 \\
\hline Surgical approach & & & 0.007 \\
\hline Laparotomy & $2(1.72 \%)$ & $11(10.09 \%)$ & \\
\hline Laparoscopic & $114(98.28 \%)$ & $98(89.91 \%)$ & \\
\hline Type of surgery & & & NA \\
\hline Debridement & $99(85.34 \%)$ & $95(87.16 \%)$ & \\
\hline Debridement + ovarian cystectomy & $10(8.62 \%)$ & $5(4.59 \%)$ & \\
\hline Debridement + myomectomy & $3(2.59 \%)$ & $2(1.83 \%)$ & \\
\hline Debridement + bilateral tubal ligation & $2(1.72 \%)$ & $6(5.50 \%)$ & \\
\hline Debridement + myomectomy + ligation & $1(0.86 \%)$ & $1(0.92 \%)$ & \\
\hline Debridement + tubal repair and orthopedic & $1(0.86 \%)$ & 0 & \\
\hline Pregnancy position & & & 0.576 \\
\hline Oviduct & $113(97.41 \%)$ & $104(95.41 \%)$ & \\
\hline Cornual & $3(2.59 \%)$ & $4(3.67)$ & \\
\hline Abdominal cavity & 0 & $1(0.92 \%)$ & \\
\hline Gestation (weeks) & $7(4,10)$ & $7(4,10)$ & 0.056 \\
\hline ASA grading & & & 0.085 \\
\hline II & $57(49.14 \%)$ & $68(62.39 \%)$ & \\
\hline III & $51(43.97 \%)$ & $38(34.86 \%)$ & \\
\hline IV & $8(6.90 \%)$ & $3(2.75 \%)$ & \\
\hline
\end{tabular}

NA: nonapplicable.

TAble 2: Operative data.

\begin{tabular}{|c|c|c|c|}
\hline Parameters & $\operatorname{ICS}(n=116)$ & Controls $(n=109)$ & $P$ value \\
\hline Operation time (min) & $81.5(38,190)$ & $80(35,222)$ & 0.393 \\
\hline Hospitalization days (days) & $5(2,9)$ & $5(3,9)$ & 0.007 \\
\hline Cure rate & $116(100 \%)$ & $109(100 \%)$ & NA \\
\hline Amount of blood loss (mL) & $2020(1210,4500)$ & $2000(1200,5000)$ & 0.105 \\
\hline Amount of autologous transfusion $(\mathrm{mL})$ & $1500(750,3000)$ & $\mathrm{NA}$ & \\
\hline Allogenic transfusion rate & $3(2.59 \%)$ & $73(66.97 \%)$ & $<0.001$ \\
\hline Infusion of allogenic coagulation factors & $9(7.76 \%)$ & $46(42.20 \%)$ & $<0.001$ \\
\hline ICU occupancy & 0 & $1(0.9 \%)$ & 0.484 \\
\hline Classification of wound healing & & & NA \\
\hline First intention & $116(100 \%)$ & $109(100 \%)$ & \\
\hline Second intention & 0 & 0 & \\
\hline Surgical complications & & & NA \\
\hline Infection & 0 & 0 & \\
\hline Wound bleeding & 0 & 0 & \\
\hline Transfusion-related adverse reactions & & & NA \\
\hline Allergy & 0 & 0 & \\
\hline Purpura & 0 & 0 & \\
\hline Nonhemolytic febrile reaction & 0 & 0 & \\
\hline Bleeding & 0 & 0 & \\
\hline Hemolysis & 0 & 0 & \\
\hline Transfusion-related acute lung injury & 0 & 0 & \\
\hline Anemia-related complications & & & NA \\
\hline Dizziness & 0 & 0 & \\
\hline Palpitation & 0 & 0 & \\
\hline Chest distress and chest pain & 0 & 0 & \\
\hline $\mathrm{Hb}$ at discharge $(\mathrm{g} / \mathrm{L})$ & $87(58,110)$ & $79(54,102)$ & $<0.001$ \\
\hline
\end{tabular}




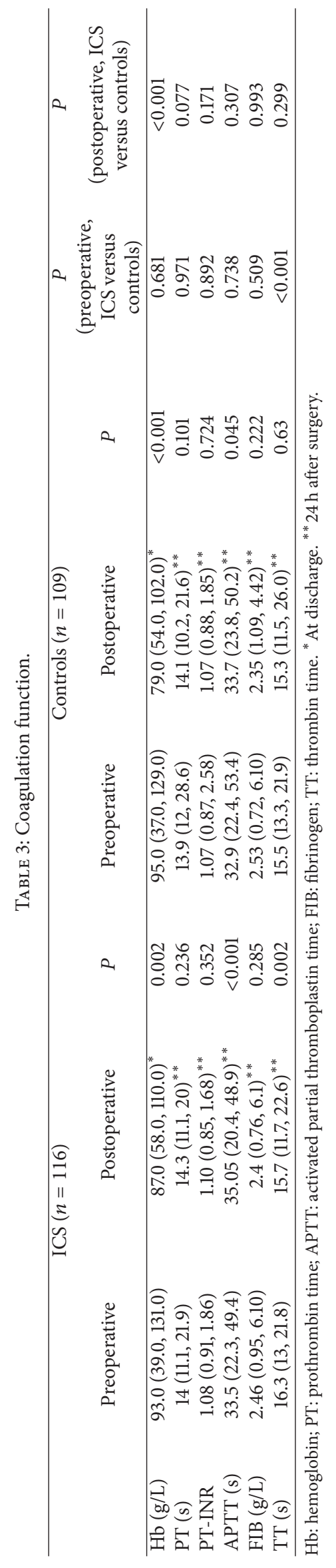


TABLE 4: Characteristics of the patients according to the amount of blood loss.

\begin{tabular}{|c|c|c|c|c|}
\hline & Blood loss (mL) & ICS $(n=116)$ & Controls $(n=109)$ & $P$ value \\
\hline \multirow{6}{*}{$n$} & $1200-1599$ & 36 & 43 & \multirow{6}{*}{0.796} \\
\hline & $1600-1999$ & 14 & 11 & \\
\hline & $2000-2399$ & 37 & 30 & \\
\hline & $2400-2799$ & 18 & 13 & \\
\hline & 2800-3199 & 7 & 8 & \\
\hline & $\geq 3200$ & 4 & 4 & \\
\hline \multirow{6}{*}{$\begin{array}{l}\text { Requirement for } \\
\text { allogenic blood } \\
\text { (cases) }\end{array}$} & $1200-1599$ & 0 & $13(30.23 \%)$ & $<0.001$ \\
\hline & 1600-1999 & $1(7.14 \%)$ & $10(90.91 \%)$ & $<0.001$ \\
\hline & $2000-2399$ & $1(2.70 \%)$ & $25(83.33 \%)$ & $<0.001$ \\
\hline & $2400-2799$ & $1(5.56 \%)$ & $13(100 \%)$ & $<0.001$ \\
\hline & $2800-3199$ & 0 & $8(100 \%)$ & $<0.001$ \\
\hline & $\geq 3200$ & 0 & $4(100 \%)$ & 0.029 \\
\hline \multirow{6}{*}{$\begin{array}{l}\text { Requirement for } \\
\text { coagulation factors } \\
\text { (cases) }\end{array}$} & $1200-1599$ & $1(2.78 \%)$ & $5(11.63 \%)$ & 0.212 \\
\hline & $1600-1999$ & $1(7.14 \%)$ & $7(63.64 \%)$ & 0.007 \\
\hline & $2000-2399$ & $2(5.41 \%)$ & $15(50.00 \%)$ & $<0.001$ \\
\hline & $2400-2799$ & $2(11.11 \%)$ & $9(69.23 \%)$ & 0.002 \\
\hline & $2800-3199$ & $2(28.57 \%)$ & $7(87.50 \%)$ & 0.041 \\
\hline & $\geq 3200$ & $1(25.00 \%)$ & $3(75.00 \%)$ & 0.486 \\
\hline \multirow{6}{*}{$\begin{array}{l}\text { Hemoglobin before } \\
\text { discharge }(\mathrm{g} / \mathrm{L})\end{array}$} & $1200-1599$ & $79.5(59,105)$ & $82(57,96)$ & 0.969 \\
\hline & $1600-1999$ & $91.5(73,109)$ & $77(60,89)$ & 0.004 \\
\hline & $2000-2399$ & $86(65,108)$ & $74(62,102)$ & $<0.001$ \\
\hline & $2400-2799$ & $92.5(58,110)$ & $76(66,92)$ & 0.001 \\
\hline & $2800-3199$ & $81(73,93)$ & $81.5(54,100)$ & 0.955 \\
\hline & $\geq 3200$ & $73(66,91)$ & $88.5(63,101)$ & 0.57 \\
\hline
\end{tabular}

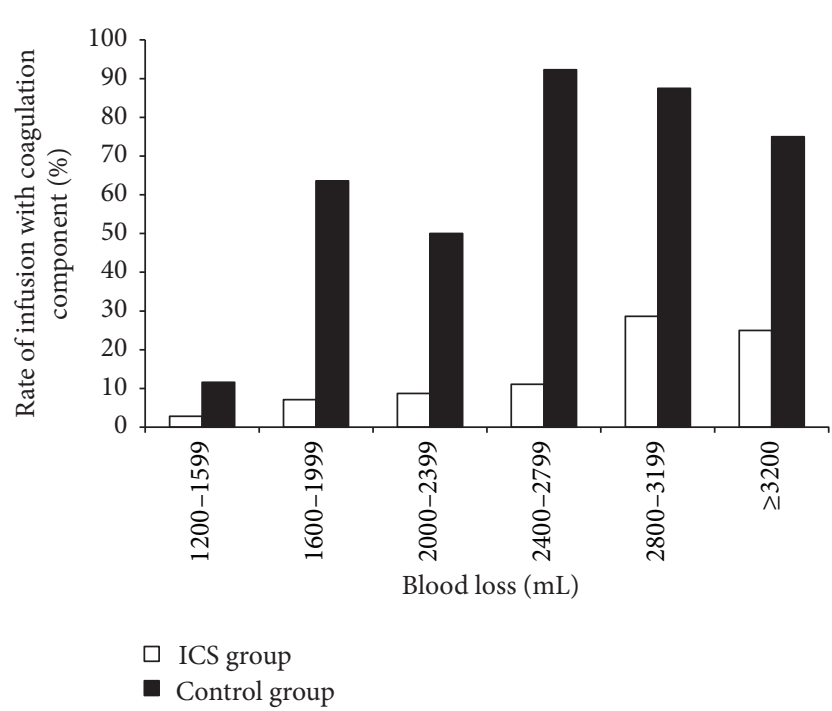

FIGURE 1: Rate of infusion of coagulation component (\%) in the ICS and control groups.

with ectopic pregnancy and severe blood loss. Therefore, the present study aimed to observe the impact of cell salvage transfusion on the coagulation function and clinical outcomes of patients with ruptured ectopic pregnancy and severe blood loss. Results suggest that ICS was associated with good clinical outcomes in patients with ruptured ectopic pregnancy and severe blood loss.

In the present study, the amount of blood loss in the ICS group was about $2300 \mathrm{~mL}$. There were only nine patients who required allogenic blood products, accounting for $7.7 \%$ of the patients, and the average amount was $400 \mathrm{~mL}$. One patient had a $4000-\mathrm{mL}$ blood loss (>90\% of the estimated blood volume) and received an autologous transfusion of $2500 \mathrm{~mL}$, $400 \mathrm{~mL}$ of plasmas, and $10 \mathrm{U}$ of cryoprecipitate. In the present study, blood loss was similar between the two groups.

Howard [4] reported that, through repeated washing, cell salvage transfusion can lead to a lack of coagulation factors, potentially leading to coagulopathy and even serious complications. Rollins et al. [5] reported one patient who received complex aortic surgery under cardiopulmonary bypass: the patient received a large amount of autologous transfusion in the operation and postoperative coagulopathy occurred; the state of the patient improved after using protamine to neutralize heparin.

North American and European studies have shown that normal blood coagulation can be achieved after cell salvage transfusion if the coagulation factors can be maintained at $20-30 \%$ of the normal levels [6]. Retrospective and prospective cohort studies, mostly performed in military trauma patients, suggested that early fresh frozen plasma transfusion 
TABLE 5: Subgroup analysis of coagulation function.

\begin{tabular}{|c|c|c|c|c|}
\hline & Blood loss (mL) & $\operatorname{ICS}(n=116)$ & Controls $(n=109)$ & $P$ (ICS versus controls) \\
\hline \multirow{6}{*}{ PT-pre (s) } & $1200-1599$ & $13.85(12.4,21.7)$ & $13.9(12,17.5)$ & 0.655 \\
\hline & $1600-1999$ & $14.05(12.7,15.6)$ & $14(12.7,15)$ & 0.851 \\
\hline & $2000-2399$ & $14.1(12.3,21.9)$ & $13.9(12.2,16.9)$ & 0.328 \\
\hline & $2400-2799$ & $13.5(11.1,18.4)$ & $13.7(12.3,16.6)$ & 0.890 \\
\hline & $2800-3199$ & $13.0(12.4,14.7)$ & $13.7(12.9,15.2)$ & 0.189 \\
\hline & $\geq 3200$ & $14.2(12.7,14.6)$ & $15.65(13,28.6)$ & 0.200 \\
\hline \multirow{6}{*}{ PT-post (s) } & $1200-1599$ & $14.7(11.7,20.0)$ & $13.9(10.3,20)$ & 0.033 \\
\hline & $1600-1999$ & $13.8(12.5,19.0)$ & $15.3(10.2,16.3)$ & 0.767 \\
\hline & $2000-2399$ & $14.3(11.2,19.8)$ & $13.85(10.7,17.2)$ & 0.097 \\
\hline & $2400-2799$ & $13.6(12.3,19.9)$ & $13.8(10.8,16.3)$ & 0.828 \\
\hline & $2800-3199$ & $14.0(11.1,15.3)$ & $14.05(11.3,16.3)$ & 0.955 \\
\hline & $\geq 3200$ & $14.2(12.8,15.2)$ & $14.35(13.2,21.6)$ & 0.686 \\
\hline \multirow{6}{*}{ PT-INR-pre } & $1200-1599$ & $1.07(0.96,1.85)^{*}$ & $1.07(0.87,1.43)$ & 0.661 \\
\hline & $1600-1999$ & $1.12(0.93,1.25)$ & $1.09(0.95,1.17)$ & 0.403 \\
\hline & $2000-2399$ & $1.09(0.91,1.86)$ & $1.07(0.89,1.35)$ & 0.492 \\
\hline & $2400-2799$ & $1.06(0.93,1.54)$ & $1.04(0.9,1.35)$ & 1.000 \\
\hline & $2800-3199$ & $0.99(0.96,1.15)$ & $1.08(0.98,1.15)$ & 0.121 \\
\hline & $\geq 3200$ & $1.11(0.95,1.14)$ & $1.23(0.97,1.58)$ & 0.200 \\
\hline \multirow{6}{*}{ PT-INR-post } & $1200-1599$ & $1.14(0.95,1.68)^{*}$ & $1.07(0.88,1.56)$ & 0.009 \\
\hline & $1600-1999$ & $1.07(0.91,1.57)$ & $1.19(0.92,1.29)$ & 0.609 \\
\hline & $2000-2399$ & $1.12(0.91,1.65)$ & $1.07(0.9,1.38)$ & 0.434 \\
\hline & $2400-2799$ & $1.02(0.9,1.67)$ & $1.04(0.89,1.29)$ & 1.000 \\
\hline & $2800-3199$ & $1.07(0.85,1.23)$ & $1.06(0.9,1.29)$ & 0.955 \\
\hline & $\geq 3200$ & $1.08(0.94,1.21)$ & $1.09(0.98,1.85)$ & 0.686 \\
\hline \multirow{6}{*}{ APTT-pre (s) } & $1200-1599$ & $33.7(23.6,49.4)^{* *}$ & $33.4(23.2,45.4)$ & 0.848 \\
\hline & $1600-1999$ & $33.8(29,48.6)$ & $33.2(26.6,45)^{\#}$ & 0.851 \\
\hline & $2000-2399$ & $33.6(26.3,44.6)$ & $32.2(22.4,46.8)$ & 0.304 \\
\hline & $2400-2799$ & $31.9(23.6,49.4)$ & $32.8(26.8,39.8)$ & 0.293 \\
\hline & $2800-3199$ & $33.0(22.3,37.9)$ & $33.6(31.6,39.8)$ & 0.779 \\
\hline & $\geq 3200$ & $33.0(31.7,40.5)$ & $30.7(29,53.4)$ & 0.486 \\
\hline \multirow{6}{*}{ APTT-post (s) } & $1200-1599$ & $35.8(28.1,45.1)^{* *}$ & $34.2(23.8,49.1)$ & 0.236 \\
\hline & $1600-1999$ & $35.2(31.2,48.9)$ & $36.2(24.3,48.1)^{\#}$ & 0.936 \\
\hline & $2000-2399$ & $34.2(28.8,47.5)$ & $33.2(27.3,42.8)$ & 0.287 \\
\hline & $2400-2799$ & $33.9(27.4,44.7)$ & $33.6(26.8,44.3)$ & 0.650 \\
\hline & $2800-3199$ & $37.0(20.4,38.8)$ & $35.2(30.2,44.2)$ & 0.779 \\
\hline & $\geq 3200$ & $33.8(29.3,44.2)$ & $35.5(28.7,50.2)$ & 1.000 \\
\hline \multirow{6}{*}{ FIB-pre (g/L) } & $1200-1599$ & $2.49(0.95,4.28)$ & $2.31(0.72,6.10)$ & 0.497 \\
\hline & $1600-1999$ & $2.24(1.59,3.95)$ & $2.69(2.12,4.17)$ & 0.107 \\
\hline & $2000-2399$ & $2.51(1.39,4.83)$ & $2.67(1.51,3.93)$ & 0.801 \\
\hline & $2400-2799$ & $2.36(1.31,3.79)$ & $2.64(1.42,3.88)$ & 0.051 \\
\hline & $2800-3199$ & $2.46(1.81,3.47)$ & $2.67(2.34,4.14)$ & 0.397 \\
\hline & $\geq 3200$ & $2.54(2.21,2.84)$ & $2.05(0.73,2.90)$ & 0.486 \\
\hline \multirow{6}{*}{ FIB-post (g/L) } & $1200-1599$ & $2.37(0.76,3.81)$ & $2.33(1.09,4.42)$ & 0.918 \\
\hline & $1600-1999$ & $2.20(1.18,3.33)$ & $2.31(1.10,3.45)$ & 0.979 \\
\hline & $2000-2399$ & $2.58(1.21,3.69)$ & $2.5(1.45,3.78)$ & 0.753 \\
\hline & $2400-2799$ & $2.29(1.07,4.2)$ & $2.42(1.75,3.56)$ & 0.489 \\
\hline & $2800-3199$ & $2.58(1.90,3.54)$ & $2.31(2.03,3.02)$ & 0.779 \\
\hline & $\geq 3200$ & $2.56(1.62,3.65)$ & $2.12(1.65,3.65)$ & 0.686 \\
\hline
\end{tabular}


TABle 5: Continued.

\begin{tabular}{ccccc}
\hline & Blood loss $(\mathrm{mL})$ & ICS $(n=116)$ & Controls $(n=109)$ & $P$ (ICS versus controls) \\
\hline & $1200-1599$ & $16.6(13.5,21.8)$ & $16.0(13.3,21.9)$ & 0.128 \\
TT-pre (s) & $1600-1999$ & $15.9(14.3,17.7)$ & $15.5(13.7,18.8)$ & 0.501 \\
& $2000-2399$ & $16.2(13.7,21.8)$ & $15.1(13.5,17.1)$ & $<0.001$ \\
& $2400-2799$ & $16.4(14.3,20.3)$ & $15.4(14.5,19)$ & 0.089 \\
& $2800-3199$ & $17.2(13,18.7)$ & $15.1(13.7,17.1)$ & 0.054 \\
& $\geq 3200$ & $16.7(14.7,19)$ & $15.6(14.7,20.3)$ & 0.886 \\
\hline & $1200-1599$ & $15.6(12.7,22.6)$ & $15.3(13.1,26)$ & 0.690 \\
TT-post (s) & $1600-1999$ & $15.3(13.5,17.8)$ & $17.2(13.7,17.9)$ & 0.647 \\
& $2000-2399$ & $16.3(13.2,19.6)$ & $15.2(11.5,17.8)$ & 0.001 \\
& $2400-2799$ & $15.6(13.1,20.6)$ & $15.1(12.4,17.6)$ & 0.312 \\
& $2800-3199$ & $13.9(11.7,18.3)$ & $15.3(14.6,19.7)$ & $16.5(14.2,18.9)$ \\
\hline
\end{tabular}

${ }^{*} P=0.032,{ }^{* *} P=0.017$, and ${ }^{\#} P=0.050$ between the two pre/post values.

with fresh frozen plasma to packed red blood cells ratio between $1: 2$ and $1: 1$ reduces 30-day mortality [13]. However, the evidence for this is of low quality and there is a lack of prospective randomized trials [13].

Ruptured ectopic pregnancy is a common gynecological problem and the patients suffer from hemorrhagic shock due to blood loss. Thus, emergency surgeries are needed. The condition of these patients is acute, dangerous, and severe, and they often require transfusion therapy. ICS transfusion could be used in ruptured ectopic pregnancy bleeding, but there is a theoretical risk of iatrogenic amniotic fluid embolism $[8,9]$. In this study, no such event was observed. Clark et al. [14] suggested that amniotic fluid is already present in normal pregnancy maternal blood and that the amount of amniotic fluid in the blood recovered after cesarean section and was lower than that of the maternal blood itself. Morikawa et al. [15] reported a study of 50 patients, in which ICS transfusion was used for hemorrhage after cesarean section; 27 patients received preoperative stored autologous transfusion at the same time. The maximum amount of recovered blood in the operation was $3715 \mathrm{~mL}$, and all patients had no transfusionrelated adverse reactions or complications due to iatrogenic amniotic fluid embolism. The National Institute for Clinical Excellence (in Britain) had recommended using leukocyte depletion filter. Many previous studies had confirmed that the application of leukocyte depletion filter could further reduce the level of amniotic fluid components in the recycled blood [16]. However, the leukocyte depletion filters could induce hypotension; thus, the prognosis of the patients may also be influenced [17]. Therefore, the physicians should be particularly cautious. The specific reason might be that the removal of leukocytes resulted in the decrease of cytokines level and the increase in the release of vasodilator substances such as bradykinin and so on [18]. A review by Tevet et al. [19] strongly suggests that the use of ICS transfusion during the perinatal period was safe. Nevertheless, there is also a theoretical risk when the mother's Rhesus is negative and that of the fetus is positive. In this study, there were no Rhesus negative women, preventing any analysis of this point. Ralph et al. [20] suggested that fetal blood may enter into the mother during pregnancy and delivery but that autologous blood containing some fetal red blood cells had no significant adverse impact on the mother. When maternal blood is Rhesus negative, the guidelines of the British Committee for Standards in Hematology (BCSH) recommend that, after infusing autologous blood, the mother should receive an intramuscular injection of anti-D immunoglobulin to prevent fetal hemolytic disease [21].

In the present study, all clinical outcomes were similar between the two groups, including cure rate, ICU occupancy, operation duration, wound healing, and complications. Hospitalization duration was significantly shorter in the ICS group, suggesting that ICS had good clinical outcomes. Priuli et al. [22] reported that, in West African countries where blood stores are minimal, cell salvage transfusion led to no adverse reactions and complications and that postoperative recovery was good. Selo-Ojeme and Feyi-Waboso [23] reported that there were no differences in the occurrence of postoperative fever and postoperative wound infection between autologous and allogenic blood transfusion and that hospitalization duration was shorter with ICS. Uchil reported that the use of ICS during cesarean section decreased the requirement for allogenic transfusion, without adverse effects [24].

The present study is not without limitations. In the calculation of blood loss, the calculation of blood clots and cotton pads was based on an empirical estimation. The selection of the surgical approach had a bias during the early stage of the study period and some patients underwent multiple procedures at the same time, which would impact operation time and hospitalization days. In the early stage of the study period, under the influence of the traditional concept that plasma infusion for acute patients with severe blood loss is helpful to improve prognosis, some physicians failed to strictly enforce the indications for plasma transfusion in the nonautologous transfusion group, which resulted in unnecessary transfusion and waste of blood products and increased the risk of allogenic transfusion. Additional studies are still necessary to confirm the safety and efficacy of ICS for patients with ruptured ectopic pregnancy and sever blood loss. 


\section{Conclusions}

ICS was associated with good clinical outcomes in patients with ruptured ectopic pregnancy and severe blood loss. These results provide some evidence for the management of blood transfusion in patients with ectopic pregnancy and severe blood loss.

\section{Conflicts of Interest}

The authors declare that there are no conflicts of interest regarding the publication of this paper.

\section{Acknowledgments}

This study was supported by the Science and Technology Fund of Nanning, Guangxi Province (no. 20123240).

\section{References}

[1] S. Kozek-Langenecker, "Management of massive operative blood loss," Minerva Anestesiologica, vol. 73, no. 7-8, pp. 401415, 2007.

[2] A. Walunj, A. Babb, and R. Sharpe, "Autologous blood transfusion," Continuing Education in Anaesthesia, Critical Care and Pain, vol. 6, no. 5, pp. 192-196, 2006.

[3] P. A. Carless, D. A. Henry, A. J. Moxey, D. O’Connell, T. Brown, and D. A. Fergusson, "Cell salvage for minimising perioperative allogeneic blood transfusion," Cochrane database of systematic reviews, vol. 4, article CD001888, 2010.

[4] L. S. Howard, "Last call for the flight simulation test?" European Respiratory Journal, vol. 42, no. 5, pp. 1175-1177, 2013.

[5] K. E. Rollins, N. L. Trim, R. J. Luddington et al., "Coagulopathy associated with massive cell salvage transfusion following aortic surgery," Perfusion, vol. 27, no. 1, pp. 30-33, 2012.

[6] G. A. Nuttall, B. C. Brost, R. T. Connis et al., "Practice guidelines for perioperative blood transfusion and adjuvant therapies: an updated report by the American society of anesthesiologists task force on perioperative blood transfusion and adjuvant therapies," Anesthesiology, vol. 105, no. 1, pp. 198-208, 2006.

[7] L. Kuppurao and M. Wee, "Perioperative cell salvage," Continuing Education in Anaesthesia, Critical Care and Pain, vol. 10, no. 4, Article ID mkq017, pp. 104-108, 2010.

[8] S. Catling and S. L. Haynes, "Coagulopathy during intraoperative cell salvage in a patient with major obstetric haemorrhage," British Journal of Anaesthesia, vol. 106, no. 5, p. 749, 2011.

[9] P. Rudra and S. Basak, "Coagulopathy during intraoperative cell salvage in a patient with major obstetric haemorrhage," British Journal of Anaesthesia, vol. 106, no. 2, pp. 280-281, 2011.

[10] R. D. Miller, L. I. Eriksson, L. A. Fleisher, J. P. Wiener-Kronish, and W. L. Young, Miller's Anesthesia, Saunders, Philadelphia, Pa, USA, 8th edition, 2015.

[11] American Society of Anesthesiologists Task Force on Perioperative Blood Management, "Practice guidelines for perioperative blood management: an update report by the American Society of Anesthesiologists Task Force on Perioperative Blood Management," Anesthesiology, vol. 122, no. 2, pp. 241-275, 2015.

[12] J. L. Carson, B. J. Grossman, and S. Kleinman, "Red blood cell transfusion: a clinical practice guideline from the AABB," Annals of Internal Medicine, vol. 157, no. 1, pp. 49-58, 2012.
[13] S. A. Kozek-Langenecker, P. Afshari, P. Albaladejo et al., "Management of severe perioperative bleeding: guidelines from the European Society of Anaesthesiology," European Journal of Anaesthesiology, vol. 30, no. 6, pp. 270-382, 2013.

[14] S. L. Clark, Z. Paylova, J. Greenspoon, J. Horenstein, and J. P. Phelan, "Squamous cells in the maternal pulmonary circulation," American Journal of Obstetrics and Gynecology, vol. 154, no. 1, pp. 104-106, 1986.

[15] M. Morikawa, A. Kuramoto, M. Nakayama et al., "Intraoperative red cell salvage during obstetric surgery in 50 Japanese women," International Journal of Gynecology and Obstetrics, vol. 128, no. 3, pp. 256-259, 2015.

[16] H. Goucher, C. A. Wong, S. K. Patel, and P. Toledo, "Cell salvage in obstetrics," Anesthesia and Analgesia, vol. 121, no. 2, pp. 465468, 2015.

[17] W. K. Rogers, S. A. Wernimont, G. C. Kumar, E. Bennett, and D. H. Chestnut, "Acute hypotension associated with intraoperative cell salvage using a leukocyte depletion filter during management of obstetric hemorrhage due to amniotic fluid embolism," Anesthesia and Analgesia, vol. 117, no. 2, pp. 449-452, 2013.

[18] D. M. Arnold, G. Molinaro, T. E. Warkentin et al., "Hypotensive transfusion reactions can occur with blood products that are leukoreduced before storage," Transfusion, vol. 44, no. 9, pp. 1361-1366, 2004.

[19] A. Tevet, S. Grisaru-Granovsky, A. Samueloff, and A. Ioscovich, "Peripartum use of cell salvage: A university practice audit and literature review," Archives of Gynecology and Obstetrics, vol. 285, no. 2, pp. 281-284, 2012.

[20] C. J. Ralph, I. Sullivan, and J. Faulds, "Intraoperative cell salvaged blood as part of a blood conservation strategy in caesarean section: is fetal red cell contamination important?" British Journal of Anaesthesia, vol. 107, no. 3, pp. 404-408, 2011.

[21] H. Qureshi, E. Massey, D. Kirwan et al., "BCSH guideline for the use of anti-D immunoglobulin for the prevention of haemolytic disease of the fetus and newborn," Transfusion Medicine, vol. 24, no. 1, pp. 8-20, 2014.

[22] G. Priuli, R. Darate, R. X. Perrin, J. Lankoande, and N. Drouet, "Multicentre experience with a simple blood salvage technique in patients with ruptured ectopic pregnancy in sub-Sahelian West Africa," Vox Sanguinis, vol. 97, no. 4, pp. 317-323, 2009.

[23] D. O. Selo-Ojeme and P. A. Feyi-Waboso, "Salvage autotransfusion versus homologous blood transfusion for ruptured ectopic pregnancy," International Journal of Gynecology and Obstetrics, vol. 96, no. 2, pp. 108-111, 2007.

[24] D. Uchil, "Cell salvage in laparoscopic surgery for ectopic pregnancy with massive hemoperitoneum," Journal of Minimally Invasive Gynecology, vol. 22, no. 6, pp. S208-S209, 2015. 


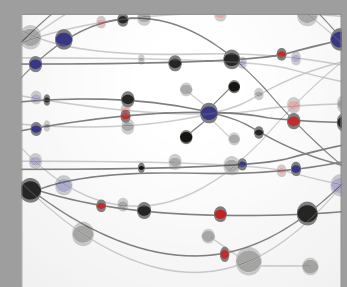

The Scientific World Journal
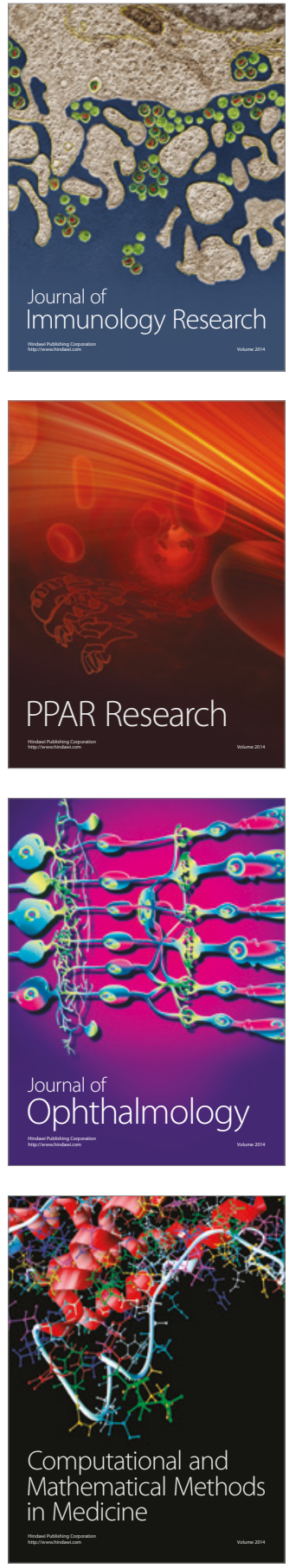

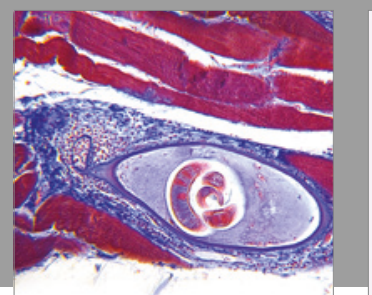

Gastroenterology Research and Practice
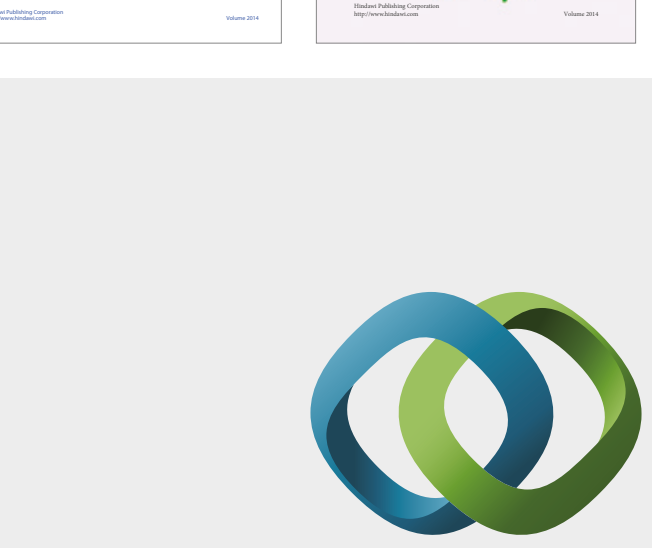

\section{Hindawi}

Submit your manuscripts at

https://www.hindawi.com
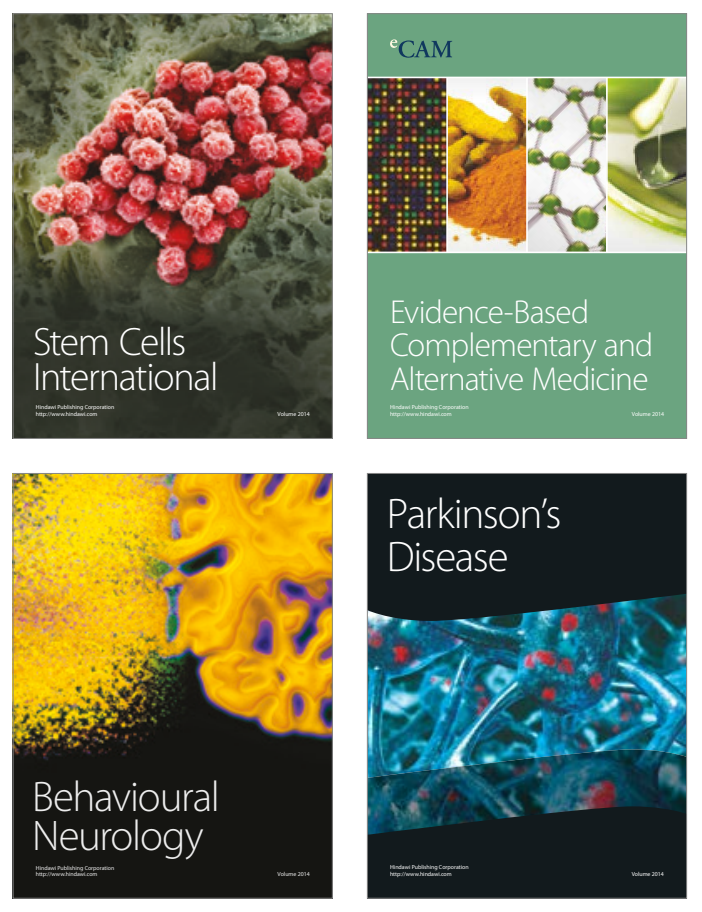
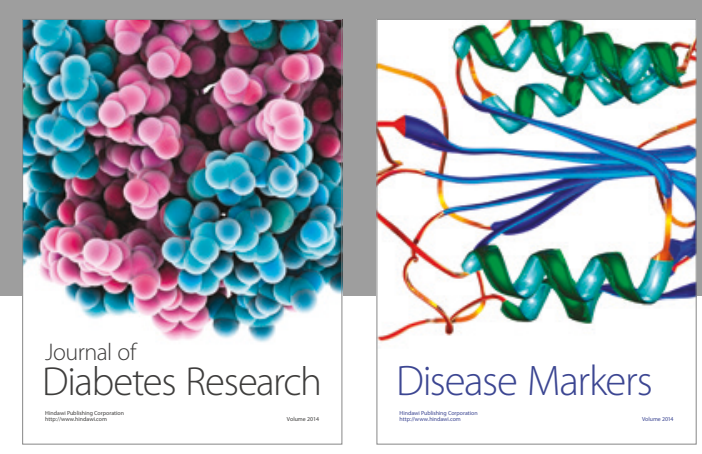

Disease Markers
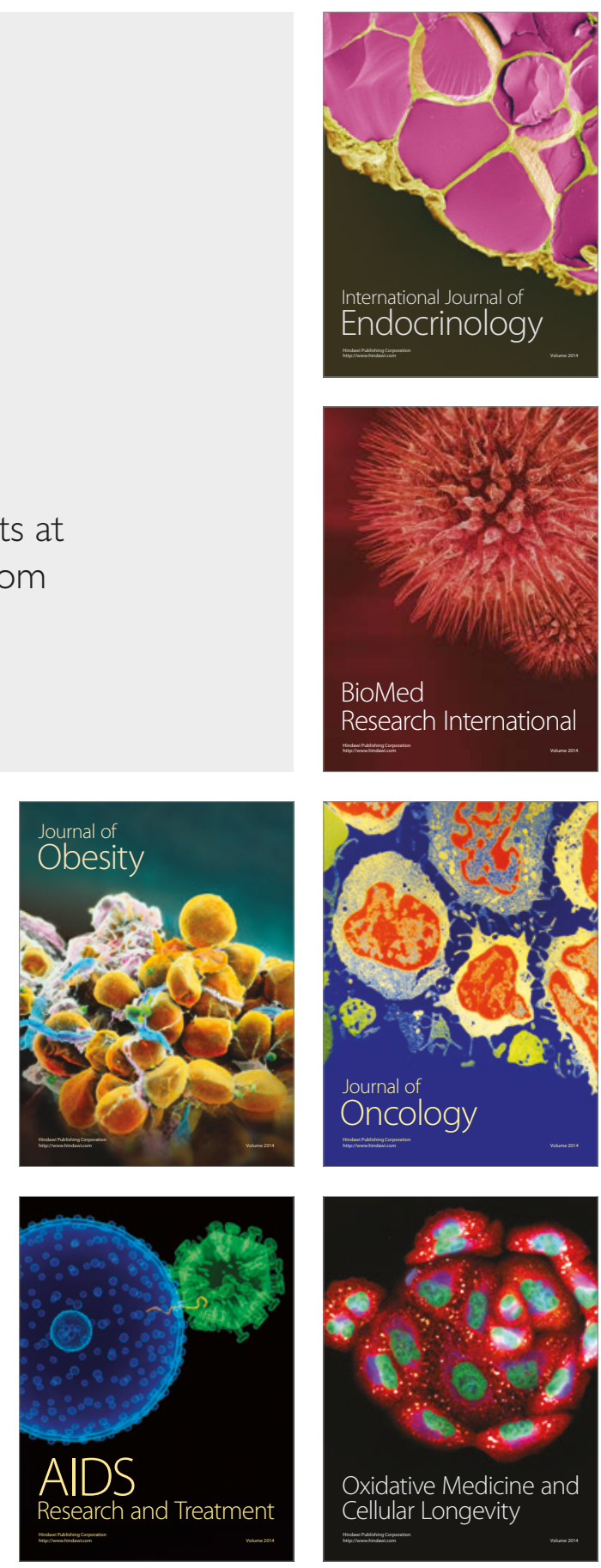